\title{
Imbalance between subpopulations of regulatory $T$ cells in COPD
}

\author{
Jia Hou, ${ }^{1,2}$ Yongchang Sun, ${ }^{1}$ Yu Hao, ${ }^{3,4}$ Jie Zhuo, ${ }^{1}$ Xiaofang Liu, ${ }^{1}$ Peng Bai, ${ }^{1}$ \\ Junyan Han, ${ }^{3,4}$ Xiwei Zheng, ${ }^{2}$ Hui Zeng ${ }^{3,4}$
}

\begin{abstract}
- Additional material is published online only. To view please visit the journal online (http://dx.doi.org/10.1136/ thoraxjnl-2012-201956).

${ }^{1}$ Department of Respiratory Medicine, Beijing Tongren Hospital, Capital Medical University, Beijing, China ${ }^{2}$ Department of Respiratory and Critical Care Medicine, General Hospital of Ningxia Medical University, Ningxia, China

${ }^{3}$ Institute of Infectious Diseases, Beijing Ditan Hospital, Capital Medical University, Beijing, China ${ }^{4}$ Beijing Key Laboratory of Emerging and Reemerging Infectious Diseases, Beijing, China
\end{abstract}

\section{Correspondence to}

Professor Hui Zeng, Institute of Infectious Diseases, Beijing Ditan Hospital, Capital Medical University, Jingshundongjie 8, Beijing 100015, China; zenghui@ccmu.edu.cn and Professor Yongchang Sun, Department of Respiratory Medicine, Beijing Tongren Hospital, Capital Medical University, No. 1 Dongjiaominxiang, Dongcheng District, Beijing 100730, China; suny@ccmu.edu.cn

Received 23 March 2012 Revised 28 April 2013 Accepted 10 May 2013 Published Online First 7 June 2013

\section{SLinked}

- http://dx.doi.org/10.1136/ thoraxjnl-2013-203878

To cite: Hou J, Sun $Y$,

Hao $Y$, et al. Thorax

2013;68:1131-1139.

\section{ABSTRACT}

Background Recent evidence indicates that human regulatory T cells (Tregs) are composed of three distinct subpopulations: $\mathrm{CD}^{2} 5^{++}$CD45RA ${ }^{+}$resting Tregs (rTregs), $\mathrm{CD}^{25^{+++}}$CD45RA ${ }^{-}$activated Tregs (aTregs), which are suppressive, and CD25 $5^{++}$CD45RA ${ }^{-}$cytokine-secreting (Fr III) cells with pro-inflammatory capacity.

Objectives To evaluate the dynamic changes in circulating and pulmonary Treg subpopulations in smokers and patients with chronic obstructive pulmonary disease (COPD), and to explore their potential roles in COPD pathogenesis.

Methods Blood samples were obtained from 57 neversmokers, 32 smokers with normal lung function and 66 patients with COPD. Bronchoalveolar lavage (BAL) samples were taken from 12 never-smokers, 12 smokers and 18 patients with COPD. The proportions of Treg subpopulations and activated CD8 T cells were evaluated using flow cytometry.

Results In peripheral blood, increased proportions of rTregs, aTregs and Fr III cells were found in smokers compared with never-smokers, whereas patients with COPD showed decreased rTregs and aTregs, and significantly increased Fr III cells compared with smokers. The changes in Treg subpopulations, with an overall decrease in the (aTreg+rTreg):(Fr III) ratio, indicated that immune homeostasis favoured inflammation and correlated with enhanced CD8 T-cell activation $(r=-0.399, p<0.001)$ and forced expiratory volume in $1 \mathrm{~s}\left(\mathrm{FEV}_{1}\right) \%$ predicted value $(\mathrm{r}=0.435, \mathrm{p}<0.001)$. The BAL (aTreg+rTreg):(Fr III) ratios displayed more robust correlations with $\mathrm{FEV}_{1} \%$ predicted value $(\mathrm{r}=0.741$, $p<0.01)$ and activation of effector $T$ cells $(r=-0.763$, $p<0.001$ ).

Conclusions The imbalance between the antiinflammatory subsets (aTreg+rTreg) and the proinflammatory subset (Fr III) of Tregs may play an important role in COPD progression.

\section{INTRODUCTION}

The principal feature of chronic obstructive pulmonary disease (COPD) is abnormal inflammatory response of the lung to inhaled noxious gases or particles, especially to tobacco smoke. ${ }^{1}$ Accumulating evidence indicates that chronic inflammation and adaptive immunity play important roles in the development and progression of COPD. $^{2-4}$ The inflammation in COPD extends beyond the lungs and includes regional lymph nodes and the systemic circulation. ${ }^{5-7}$ However, the regulation of inflammation in patients with COPD has not yet been fully elucidated.
Key messages

What is the key question?

- Are phenotypically and functionally different subpopulations of regulatory T cells (Tregs) varied in chronic obstructive pulmonary disease (COPD) and potentially involved in the progression of inflammation?

\section{What is the bottom line?}

- The imbalance between the anti-inflammatory subsets and the pro-inflammatory subset of Tregs correlates with loss of lung function and immune activation in patients with COPD.

\section{Why read on?}

- This study provides evidence to support disturbed homeostasis of Treg subpopulations as a potential mechanism for persistent inflammation mediated by CD8 cells in COPD.

T lymphocytes are believed to be key cells in regulating airway inflammation in COPD. ${ }^{7}$ Activated T cells, especially activated CD8 T cells, can cause a variety of the tissue injuries that typify COPD by direct cytopathic effects, elaboration of diverse pro-inflammatory and deleterious mediators, and/or recruitment and activation of other immune and parenchymal effector cells. ${ }^{8}{ }^{9} \mathrm{~A}$ number of studies have demonstrated that activation of $T$ cells is tightly controlled by active mechanisms and negative regulatory mechanisms. In the past decade, $\mathrm{CD} 4^{+} \mathrm{CD} 25^{+} \mathrm{Foxp}^{+}{ }^{+}$Tregulatory cells (Tregs), a critical subset of $\mathrm{T}$ cells that use a variety of mechanisms to suppress the immune response, have gained considerable attention, ${ }^{10-14}$ and Treg abnormalities have been described in many chronic inflammatory and autoimmune disorders. ${ }^{10}{ }^{11}$ Recently, accumulating evidence indicates that patients with COPD exhibit many of the characteristics of a classical autoimmune response..$^{15} 16$ For these reasons, several studies have evaluated the role of Tregs in adaptive immunity of COPD. In patients with COPD, Treg proportions fluctuate in bronchoalveolar lavage (BAL) or in different parts of pulmonary tissue. ${ }^{17-20}$ However, contrary to the current notion of systemic inflammation, the frequencies of Tregs in peripheral blood do not differ significantly among patients with COPD, healthy smokers and never-smokers. ${ }^{17-20}$ 
In most previous studies, Tregs have only been considered as a whole population. However, recent studies have demonstrated that human $\mathrm{CD}^{+}{ }^{+} \mathrm{CD} 25^{+}$Foxp3 ${ }^{+}$Tregs are heterogeneous and comprise three distinct subpopulations, each with a precise phenotype and function. Apart from resting Tregs (rTregs, CD25 $5^{++}$ $\mathrm{CD} 4 \mathrm{RA}^{+}$) and activated Tregs (aTregs, CD25 ${ }^{+++} \mathrm{CD}^{+} \mathrm{RA}^{-}$), which are suppressive in vitro, human $\mathrm{CD} 4^{+} \mathrm{CD} 25^{+} \mathrm{Foxp}^{+}$ Tregs also possess CD25 ${ }^{++}$CD45RA ${ }^{-}$cytokine-secreting T cells (Fr III) with pro-inflammatory capacity. ${ }^{21}$ This population lacks suppressive activity or has limited suppressive activity, but can produce interleukin (IL)-2, IL-17 and interferon (IFN)- $\gamma,{ }^{21-25}$ which are important for T-cell activation and pulmonary inflammation. ${ }^{26}{ }^{27}$ Recent studies revealed the clinical relevance of subpopulations of Tregs in patients with autoimmune diseases, ${ }^{21} \mathrm{HIV}$ infection $^{22}$ and diabetes. ${ }^{23}$

The aim of this study was to explore whether there were abnormal distributions in the suppressive and/or the pro-inflammatory subpopulations of Tregs in the blood and the lung of patients with COPD and smokers by using this new identification strategy of Foxp $3^{+}$T-cell subsets, and whether the frequency of different Treg subsets was correlated with effector T-cell (CD8) activation and disease severity as defined by forced expiratory volume in $1 \mathrm{~s}\left(\mathrm{FEV}_{1}\right)$. The findings should provide new insights into the potential mechanisms underlying persistent inflammation and adaptive immunity in COPD.

\section{MATERIALS AND METHODS Study subjects}

Sixty-six patients with COPD and 34 smokers with normal lung function were recruited in Beijing Tongren Hospital, Capital Medical University, China. Fifty-seven never-smokers with normal lung function were also recruited as controls. Written informed consent was obtained and the local research ethics committee approved this study (TRECKT 2008-14). Subject baseline characteristics are summarised in table 1 . A total of 112 patients with COPD were consecutively invited but 46 declined.

Subjects with a smoking history of $\geq 20$ pack-years and normal lung function $\left(\mathrm{FEV}_{1}>80 \%\right.$ predicted) were categorised as smokers. COPD diagnosis was established according to the definition supplied by the Global Initiative for Chronic Obstructive Lung Disease (GOLD) guidelines. ${ }^{28}$ Patients with COPD had $\mathrm{FEV}_{1} /$ forced vital capacity ratio $<70 \%$ and $\mathrm{FEV}_{1}<80 \%$ of the predicted value post bronchodilators. All patients with COPD were clinically stable and had not experienced any exacerbations for $\geq 3$ months preceding inclusion in the study. Participants with neoplasm, interstitial lung disease,

Table 1 Demographics and spirometry of all participants

\begin{tabular}{|c|c|c|c|}
\hline & Never-smokers & Smokers & $\begin{array}{l}\text { Patients with } \\
\text { COPD }\end{array}$ \\
\hline Subjects & $n=57$ & $n=32$ & $n=66$ \\
\hline Age (year) & $64.4 \pm 4.3$ & $66.3 \pm 2.7$ & $68 \pm 3.5$ \\
\hline Men/women & $48 / 9$ & $30 / 2$ & $56 / 10$ \\
\hline Current/ex-smokers & $0 / 0$ & $26 / 6$ & $18 / 48$ \\
\hline Smoking history pack (year) & 0 & $37(32-47)$ & $40(36-48)$ \\
\hline $\mathrm{FEV}_{1} \%$ predicted & $94.5 \pm 5.5$ & $93.5 \pm 2.9$ & $47.0 \pm 8.6$ \\
\hline $\mathrm{FEV}_{1} / \mathrm{FVC} \%$ & $75.2 \pm 3.0$ & $73.8 \pm 3.9$ & $43.4 \pm 5.6$ \\
\hline Inhaled corticosteroid use & 0 & 0 & $n=26$ \\
\hline
\end{tabular}

autoimmune disease, diabetes, infection or other immunerelated diseases were excluded.

BAL fluid samples were obtained from 18 patients with COPD, 12 smokers and 12 never-smokers. The reasons for bronchoscopy were haemoptysis (COPD, $\mathrm{n}=9$ of 18 ; smokers, $\mathrm{n}=5$ of 12 ; never-smokers, $\mathrm{n}=7$ of 12 ); unexplained shortness of breath or weight loss (COPD, $\mathrm{n}=5$ of 18 ; smokers, $\mathrm{n}=3$ of 12 ; never-smokers, $n=3$ of 12 ); and investigation of abnormal chest radiograph findings (COPD, $n=4$ of 18 ; smokers, $n=4$ of 12; never-smokers, $\mathrm{n}=2$ of 12 ).

\section{Cell collection}

Peripheral blood samples were collected in ethylenediaminetetraacetic acid treated tubes from each subject and were processed to measure peripheral blood mononuclear cells (PBMCs) for flow cytometry setup procedures. Blood samples were layered onto Ficoll-Paque Plus (Amersham Biosciences, Amersham, Bucks, UK), centrifuged ( $400 \mathrm{~g}$ for $20 \mathrm{~min}$ at $21^{\circ} \mathrm{C}$ ), and PBMCs were harvested. Cells were washed once in divalent cation-free Hanks balanced salt solution at $300 \mathrm{~g}$ for $5 \mathrm{~min}$ at $4^{\circ}$ C. PBMCs were resuspended and viable counts obtained.

BAL was performed and processed as previously described. ${ }^{29}$ Bronchoscopy was performed with a flexible fibreoptic bronchoscope (Olympus, Tokyo, Japan) under topical lidocaine. Four separate $50 \mathrm{~mL}$ aliquots of sterile $0.9 \%$ saline solution were instilled in one pulmonary segment of a right middle lobe, or a lobe not affected by radiographic or endobronchial abnormalities. The fluid recovered was filtered, washed twice in phosphate-buffered saline and resuspended at $10^{6}$ cells $/ \mathrm{ml}$ in RPMI-1640 medium at $4^{\circ} \mathrm{C}$. BAL fluid and peripheral blood samples were processed immediately after attainment.

\section{Flow cytometry}

Freshly obtained human PBMC and BAL samples were stained as previously described ${ }^{21}$ with anti-hCD4-FITC (e-Bioscience, San Diego, California, USA), anti-hCD25-PE-Cy5 (BD Biosciences, San Jose, California, USA), anti-hCD45RA-APC (BD Biosciences). Anti-hCD8-FITC, anti-hCD28-APC, antihCD38-APC, anti-hHLA-DR-PE and anti-hCD69-FITC (BD Biosciences) antibodies were used for surface marker staining of each subpopulations. Treg subpopulations were gated as shown in online supplementary figure S1. Details of the cell counting, intracellular staining, cell culture and in vitro suppression assay are provided in the online supplementary appendix.

\section{Data analysis}

Group data were expressed as mean and SEM or as median and IQR when appropriate. For data not distributed normally, comparisons between three groups were made using a one-way Kruskal-Wallis test $(\mathrm{p}<0.05$ was considered statistically significant). If this test indicated significance, the Mann-Whitney test was used for post hoc analysis for comparison between two groups, with corrections of $\mathrm{p}$ values according to Bonferroni $(p<0.017$ was considered statistically significant). Correlation was assessed by calculating Spearman's rank correlation coefficient. Here, $\mathrm{p}<0.05$ was considered statistically significant. Statistical analysis was performed using SPSS for Windows V.16.0 (Chicago, Illinois, USA).

\section{RESULTS}

\section{Demographic characteristics of study population}

The characteristics of the patients with COPD, smokers and never-smokers with normal lung function are summarised in table 1 . There was no difference between the groups in terms of 
Table 2 Demographics and spirometry of participants who underwent bronchoscopy

\begin{tabular}{|c|c|c|c|}
\hline & Never-smokers & Smokers & $\begin{array}{l}\text { Patients with } \\
\text { COPD }\end{array}$ \\
\hline Subjects & $n=12$ & $n=12$ & $n=18$ \\
\hline Age (year) & $63.3 \pm 3.9$ & $65.8 \pm 2.8$ & $66.5 \pm 4.2$ \\
\hline Men/women & $10 / 2$ & $10 / 2$ & $14 / 4$ \\
\hline Current/ex-smokers & $0 / 0$ & $9 / 3$ & $8 / 10$ \\
\hline Smoking history pack (year) & 0 & $34(30-41)$ & $38(20-53)$ \\
\hline $\mathrm{FEV}_{1} \%$ predicted & $94.2 \pm 4.8$ & $92.6 \pm 3.4$ & $46.8 \pm 7.4$ \\
\hline $\mathrm{FEV}_{1} / \mathrm{FVC} \%$ & $75.2 \pm 1.4$ & $73.3 \pm 3.3$ & $42.4 \pm 5.6$ \\
\hline Inhaled corticosteroid use & 0 & 0 & $n=6$ \\
\hline
\end{tabular}

age. The unequal sex ratio was mostly due to the much higher prevalence of COPD in men than in women in China. ${ }^{30}$ There was no significant difference in the smoking history of patients with COPD and smokers. Table 2 shows the main clinical and functional characteristics of participants who underwent bronchoscopy. The demographic characteristics of this study population were consistent with those who did not receive bronchoscopy.

\section{Dissection of Foxp $3^{+}$cells into three subsets with different function and phenotypic characteristics}

As described previously, ${ }^{21} \mathrm{CD}^{+}{ }^{+}$Foxp $3^{+} \mathrm{T}$ cells were separated into three subpopulations by the expression of CD25 and CD45RA: rTregs $\left(C D 45 \mathrm{RA}^{+} \mathrm{CD} 25^{++}\right.$, Fr I), aTregs (CD45RA ${ }^{-}$ $\mathrm{CD} 25^{+++}$, Fr II) and cytokine-secreting subset $\left(\mathrm{CD} 45 \mathrm{RA}^{-}\right.$ $\mathrm{CD} 25^{++}$, Fr III) (figure 1A). As shown in figure 1A, the degrees of FoxP3 expression in these fractions were proportional to CD25 expression. Moreover, rTregs expressed high levels of CD31, a marker for primary thymic emigrants, but expression was lost during their post-thymic peripheral expansion, as reported previously. ${ }^{21}{ }^{31}$ Compared with rTregs, aTregs showed lower CD31 expression but higher expression of Foxp3, cytotoxic T-lymphocyte antigen 4 (CTLA-4) and HLA-DR, and $\mathrm{Ki}-67$, a nuclear protein expressed in proliferating cells (figure $1 \mathrm{~B})$. These data suggest that these cells are a proliferating, activated fraction of Tregs. Although Fr III cells expressed intermediate levels of Foxp3, decreased levels of CTLA-4, HLA-DR and Ki-67 were observed. Meanwhile, a higher amount of IL-17 and IFN- $\gamma$ were detected in response to phorbol-12myristate-13-acetate (PMA) and calcium ionomycin stimulation (figure 1C), which confirmed that this subpopulation represented previously described pro-inflammatory cytokine-secreting cells. $^{21}$

\section{Varied subpopulation frequencies of circulating Tregs in patients with COPD, smokers and never-smokers}

We first investigated the frequencies of Tregs in peripheral blood of different groups using the classic definition. ${ }^{32}$ In line with previous studies, ${ }^{17}$ Treg identification using $\mathrm{CD} 4{ }^{+} \mathrm{CD} 25^{+}$ Foxp $3^{+}$showed no difference in the circulating Treg number in patients with COPD (median 8.69\%) compared with smokers (median 8.23\%, $\mathrm{p}=0.45$ ) and never-smokers (median 7.95\%, $\mathrm{p}=0.16)$. However, the proportion of $r \operatorname{Treg}(\mathrm{Fr} \mathrm{I})$, aTreg (Fr II) and cytokine-secreting (Fr III) fractions among CD4 T cells increased slightly in smokers compared with never-smokers (median, Fr I: $5.18 \%$ vs 3.25\%, p $<0.001$; FrII: $2.01 \%$ vs $1.70 \%, \mathrm{p}<0.01$; Fr III: $7.37 \%$ vs $4.78 \%, \mathrm{p}<0.001$, for smokers and never-smokers, respectively). This suggested that tobacco smoke resulted in anti-inflammatory and inflammatory responses. Strikingly, in comparison to smokers, patients with COPD showed significantly increased proportions of Fr III cells (median 10.81\%, $\mathrm{p}<0.001$ ) but decreased proportions of rTregs (median 2.16\%, p<0.0001) and aTregs (median 1.07\%, $\mathrm{p}<0.0001$; figure $2 \mathrm{~A}-\mathrm{C}$ ). The number of each subset per microlitre of peripheral blood showed the same trend of variation as the proportion of each subset in different groups (figure 2D-F). Varied subpopulation frequencies suggested that the balance between immunosuppressive subsets and inflammatory subsets in $\mathrm{CD}^{+}{ }^{+}$Foxp $^{+} \mathrm{T}$ cells shifted towards inflammation in patients with COPD.

Next, we performed in vitro experiments to test the immune function of each population in patients with COPD. As shown in figure 3A, both rTregs and aTregs were functionally suppressive, while FrIII cells showed moderate suppressive capacity and could produce IL-17 and IFN- $\gamma$ in response to PMA/ionomycin (figure 3B,C). In accordance with the phenotype results, compared with never-smokers, the numbers of Foxp $3^{+}$IL-17secreting FrIII cells and Foxp $3^{+}$IFN- $\gamma$-secreting FrIII cells were slightly increased in smokers, but dramatically increased in patients with COPD (figure 3E, F). More importantly, the number of Foxp $3^{+}$IL-17-secreting FrIII cells strongly correlated with $\mathrm{T}$ helper 17 (Th17) cells (Foxp3 $\left.{ }^{-} \mathrm{IL}_{17}{ }^{+}\right) \quad(\mathrm{r}=0.818$, $\mathrm{p}<0.001$; figure $3 \mathrm{G})$.

\section{Disturbed homeostasis among subpopulations of circulating Tregs correlated with effector T-cell activation status and disease severity}

To illustrate further the balance between the suppressive and pro-inflammatory subpopulations, we calculated the ratio of (aTreg+rTreg):(Fr III). As expected, the (aTreg+rTreg):(Fr III) ratio was significantly decreased in patients with COPD (median 0.47) compared with smokers (median 0.77; p<0.0001) and never-smokers (median $0.75 ; \mathrm{p}<0.0001$ ) (figure $4 \mathrm{~A}$ ). No difference was found in the (aTreg+rTreg):(Fr III) ratio between the patients receiving and those not receiving inhaled corticosteroids (see online supplementary figure S2), which indicated that the differences in Treg subpopulations among never-smokers, smokers and patients with COPD were not due to corticosteroids. However, a definite conclusion could only be made by a prospective study to explore specifically the effect of corticosteroids on Tregs.

It has been previously demonstrated that patients with COPD exhibited enhanced CD8 T-lymphocyte activation, which was correlated with the disease severity as measured by $\mathrm{FEV}_{1}{ }^{33}$ Moreover, it has been shown that CD8 T-cell activation could be suppressed by Tregs $^{10}$ and promoted by IL-17. ${ }^{34}$ The decreased (aTreg+rTreg):(Fr III) ratio in patients with COPD prompted us to test whether the disturbed Treg homeostasis had any influence on CD8 T-cell activation and diseases severity. The expression of CD28 (a costimulatory molecule), CD69 (an early marker transiently expressed on activated T cells), CD38 and HLA-DR (more general markers of T-cell activation) on CD8 T cells was evaluated. Similar to a previous study, ${ }^{33}$ patients with COPD showed elevated frequencies of $\mathrm{CD}^{+}{ }^{\mathrm{HLA}-\mathrm{DR}^{+}}$(median 10.72\%, 5.33\% and 4.98\% for patients with COPD, smokers and never-smokers, respectively, $\mathrm{p}<0.001$ ) and $\mathrm{CD}^{+} \mathrm{CD}^{+} 8^{+}$cells (median $14.95 \%, 8.40 \%$ and $8.24 \%$ for patients with COPD, smokers and never-smokers, respectively, $\mathrm{p}<0.001$ ) (figure $4 \mathrm{~B}, \mathrm{C}$ ). However, expression of 
A
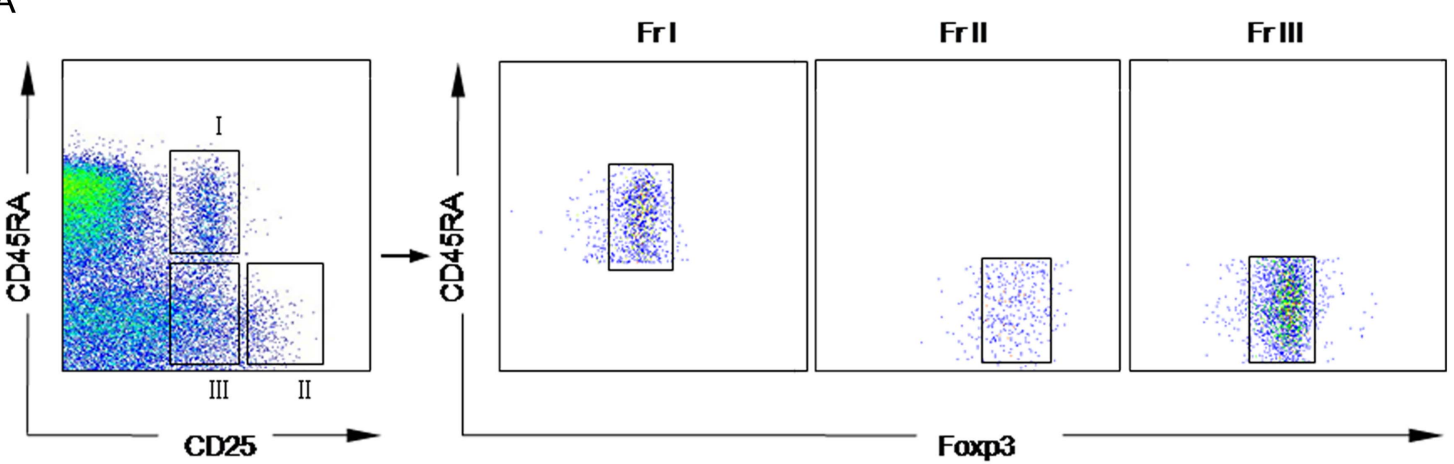

B
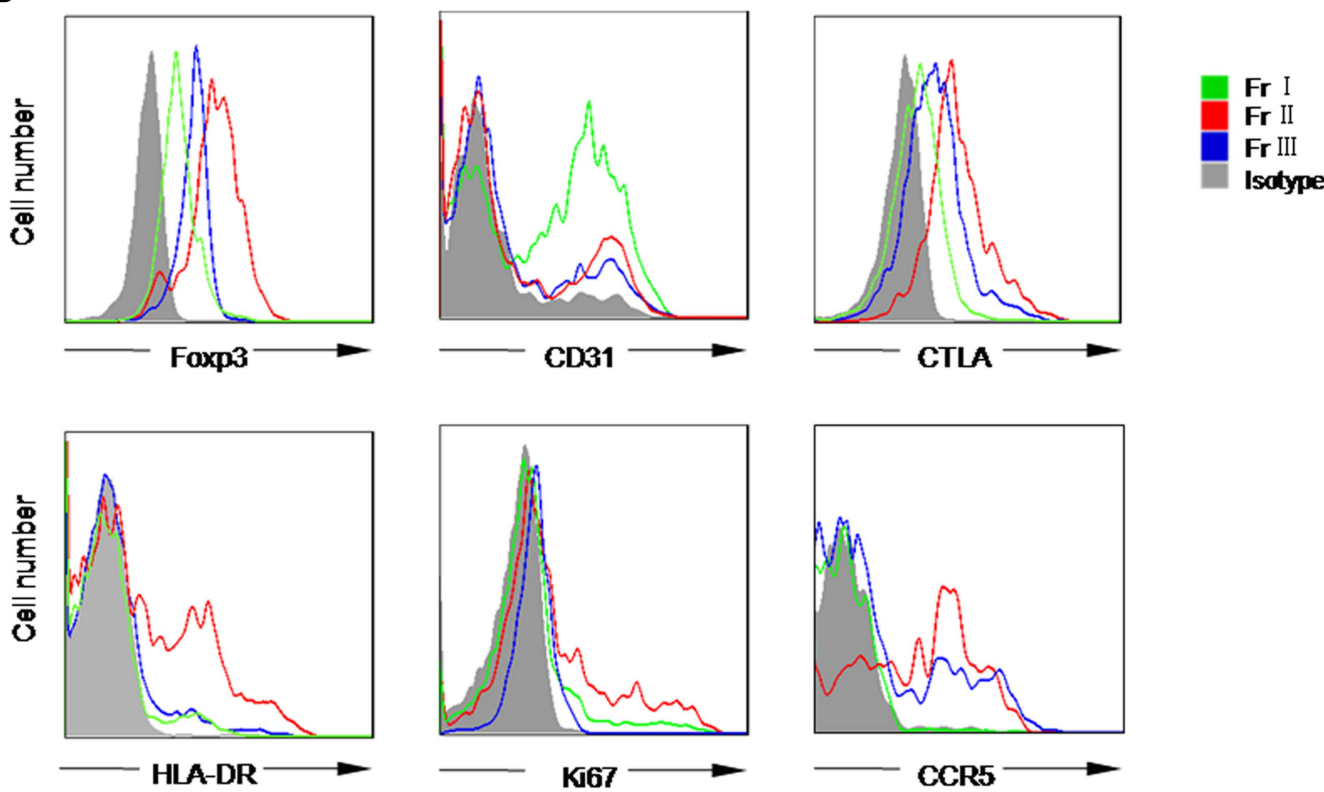

C
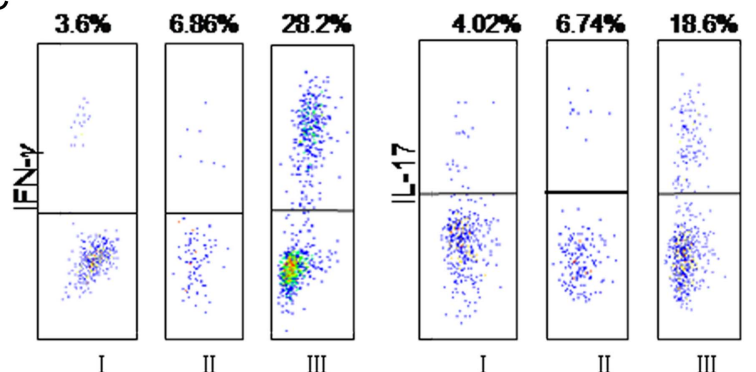

Figure 1 Dissection of $\mathrm{CD}^{+}{ }^{+}$Foxp3 ${ }^{+} \mathrm{T}$ cells into three subsets by cell surface molecules and intracellular protein. (A) Three subsets of CD4 T cells defined by the expression of CD25, CD45RA (left panel). Right panels showed the FoxP3 expression in each subpopulation. (B) Each subpopulation expressed different amounts of Foxp3, cytotoxic T-lymphocyte antigen 4 (CTLA-4), CD31, HLA-DR, CD95 and Ki67. Fr I is represented by green lines, Fr II by red lines, Fr III by blue lines and isotype by solid grey profiles.(C) Flow cytometry of the production of interleukin 17 (IL-17) and interferon $\gamma$ (IFN- $\gamma$ ) by three subsets after stimulation with phorbol-12-myristate-13-acetate (PMA) and ionomycin for $5 \mathrm{~h}$. Percentages of cytokine-secreting cells are shown. Data are representative of five independent experiments. Fr I, CD25 ${ }^{++}$CD45RA $^{+}$resting T-regulatory cells; Fr II, CD25 ${ }^{+++} \mathrm{CD}_{5} 5 \mathrm{RA}^{-}$ activated T-regulatory cells; Fr III, CD25 ${ }^{++}$CD45RA ${ }^{-}$cytokine-secreting cells.

CD28 and CD69 in CD8 T lymphocytes did not differ significantly between the groups (data not shown).

In line with previous reports that Tregs suppress CD8 T-cell activation, we found that the (aTreg+rTreg):(Fr III) ratios were inversely correlated with the frequencies of $\mathrm{CD} 8^{+} \mathrm{HLA}^{-} \mathrm{DR}^{+}$ and $\mathrm{CD}^{+} \mathrm{CD}^{+} 8^{+}$cells $(\mathrm{r}=-0.399, \mathrm{p}<0.001 ; \mathrm{r}=-0.416$, $\mathrm{p}<0.001$; figure 4E,F). More importantly, the (aTreg+rTreg):(Fr III) ratios were significantly correlated with $\mathrm{FEV}_{1}$ predicted values $(r=0.435, p<0.001$; figure $4 D)$, which indicated that the imbalance between suppressive subpopulations and the cytokine-secreting population was correlated with effector T-cell activation and severity of COPD.

\section{Disturbed homeostasis among subpopulations of Tregs in BAL}

To clarify the homeostatic status of local Tregs, BAL samples were obtained from 12 never-smokers, 12 smokers and 18 
A

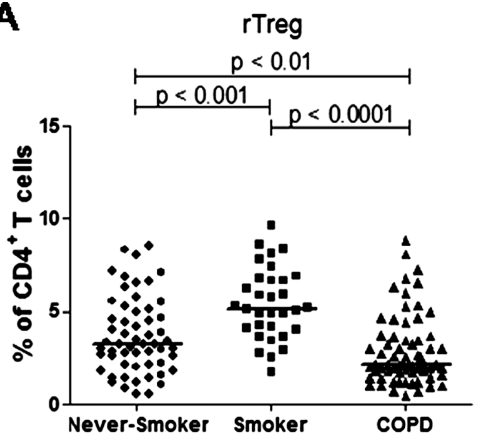

B

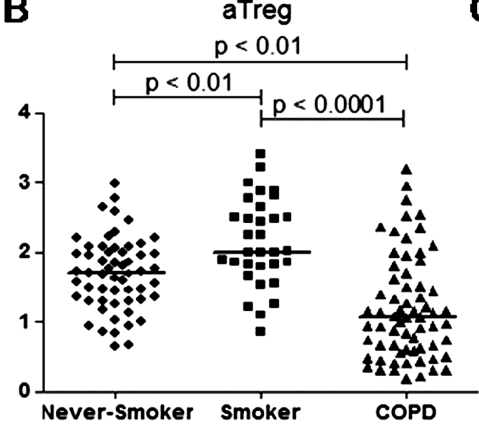

C

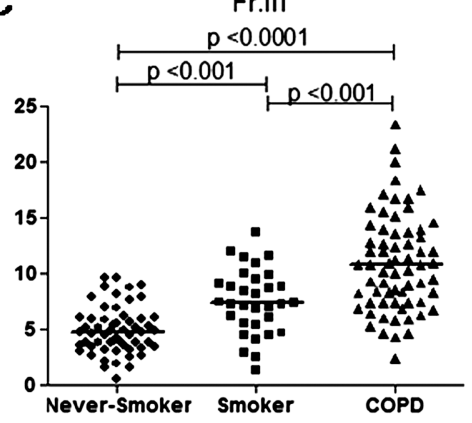

D

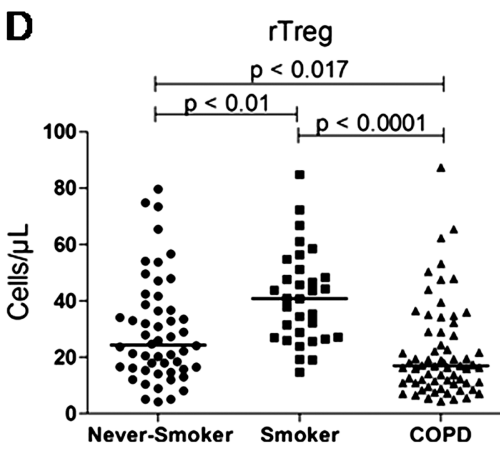

G

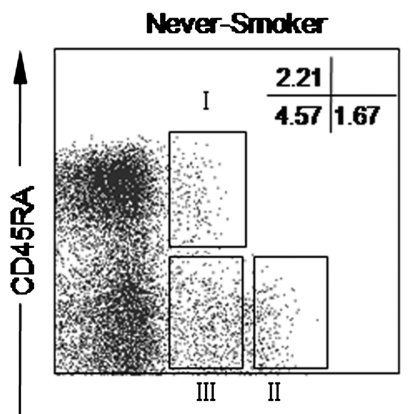

E

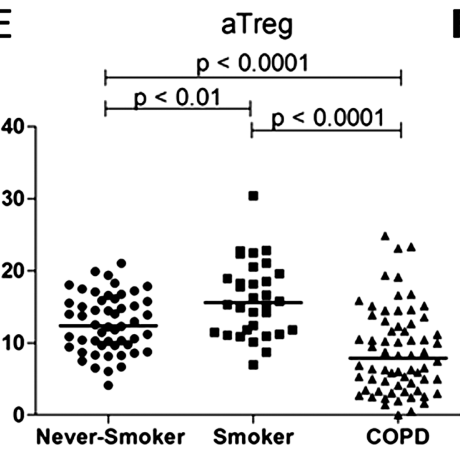

F

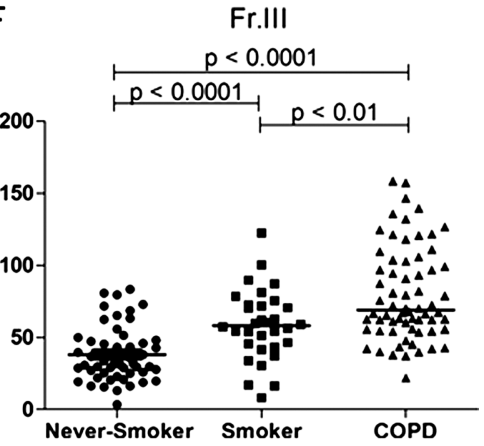

Figure 2 Variations in Foxp $3^{+}$cell subpopulations under physiological and disease conditions. (A-C) The percentages of each circulating T-regulatory subset among CD4 T cells in never-smokers, smokers and patients with chronic obstructive pulmonary disease (COPD). (D-F) Number of each Foxp3 ${ }^{+}$subset per microlitre of peripheral blood in never-smokers, smokers and patients with COPD. (G) Representative data of flow cytometry profiles are shown. Horizontal lines indicate median values. A p value $<0.017$ was considered statistically significant. $\mathrm{Fr} I, \mathrm{CD}_{2} 5^{++} \mathrm{CD} 45 \mathrm{RA}{ }^{+}$resting T-regulatory cells (rTreg); Fr II, CD25+++ CD45RA- activated T-regulatory cells (aTreg); Fr III, CD25 ${ }^{++}$CD45RA $^{-}$cytokine-secreting cells.

patients with COPD who were undergoing clinically investigational bronchoscopy. In line with a previous study, ${ }^{18}$ we found that the majority of Tregs in BAL were CD45RA-. Accordingly, the percentage of rTregs in BAL was decreased markedly compared with that in peripheral blood and was identical across all three groups. However, aTregs and FrIII cells in BAL had a similar pattern of variation in peripheral blood, that is, aTregs decreased whereas Fr III cells increased in patients with COPD compared with never-smokers and smokers (figure 5A). Next, we calculated the (aTreg+rTreg):(Fr III) ratios in BAL to illustrate the local balance between the suppressive and pro-inflammatory subpopulations. Similar to the results from circulating Tregs, we found that (aTreg+rTreg):(Fr III) ratios were significantly lower in BAL of patients with COPD compared with smokers and never-smokers, suggesting that disturbed Treg homeostasis was also present in the lung (figure 5B). More importantly, compared with the blood (aTreg+rTreg):(Fr III) ratios, the BAL ratios showed a stronger correlation with activation status of effector
T cells $\left(\mathrm{CD}^{+}{ }^{+} \mathrm{HLA}^{-D R^{+}}: \mathrm{r}=0.763, \mathrm{p}<0.001 ; \mathrm{CD}^{+} \mathrm{CD} 69^{+}\right.$ cells; $\mathrm{r}=0.713, \mathrm{p}<0.001)$ and $\mathrm{FEV}_{1} \%$ predicted value $(\mathrm{r}=0.741$, $\mathrm{p}<0.001$; figure 5C-E).

\section{DISCUSSION}

$\mathrm{CD}^{+}{ }^{+}$Foxp $^{+}$Tregs have been recognised as a negative regulatory population during immune responses. ${ }^{10} 11 \quad 12$ However, phenotypic and function heterogeneity of human $\mathrm{CD}^{+}$ Foxp $3^{+}$Tregs is a major obstacle for understanding their clinical relevance. Dissecting CD ${ }^{+}$Foxp ${ }^{+}$cells into subsets, especially defining the cytokine-producing population (Fr III), is one of the striking findings of the studies on human $\mathrm{CD}^{+}{ }^{+} \mathrm{Foxp}^{+}$ Treg heterogeneity. ${ }^{21} 2425$ Previous studies which identified Tregs as a whole did not find any significant difference in frequencies of circulating Tregs in patients with COPD. ${ }^{17}{ }^{18}$ Here, based on this new definition of Treg subsets, we revealed significantly decreased proportions of rTregs and aTregs and an increased proportion of the cytokine-secreting non-Treg 
Figure 3 In vitro functional activity of the Foxp $3^{+}$cell subpopulation in patients with chronic obstructive pulmonary disease (COPD).

(A) Suppression of T-cell proliferation by each subpopulation was assessed by carboxyfluorescein succinimidyl ester (CFSE) staining. Percentages of dividing cells were indicated. Data are representative of five independent experiments. (B and C) Intracellular staining of cytokines. Representative staining of intracellular Foxp3 versus interleukin 17 (IL-17) or interferon $\gamma$ (IFN- $\gamma)$ in never-smokers, smokers and patients with COPD after $5 \mathrm{~h}$ of stimulation with phorbol-12-myristate13-acetate (PMA)/ionomycin and GolgiStop. Data are representative of 51 independent experiments. (D-F) Frequencies of Foxp3 ${ }^{-} \mathrm{IL}_{-17^{+}}$(D), Foxp3 $^{+} \mathrm{IL}^{+} 7^{+}$(E) and Foxp3 ${ }^{+}$IFN- $\gamma$ (F) among CD4 T cells in three groups. A $p$ value $<0.017$ is considered statistically significant. The top bar in $(D-F)$ is for the difference between the never-smokers and patients with COPD. (G) Proportions of IL-17-secreting T-regulatory cells correlated with T helper 17 cells $\left(\right.$ Foxp3 $\left.3^{-} \mathrm{IL} 17^{+}\right)$. A $p$ value $<0.05$ was considered statistically significant. Fr I, $\mathrm{CD}^{2} 5^{++} \mathrm{CD}_{4} 5 \mathrm{RA}^{+}$resting T-regulatory cells; Fr II, CD25 ${ }^{+++}$CD45RAactivated T-regulatory cells; Fr III, CD25 ${ }^{++}$CD45RA $^{-}$cytokine-secreting cells.
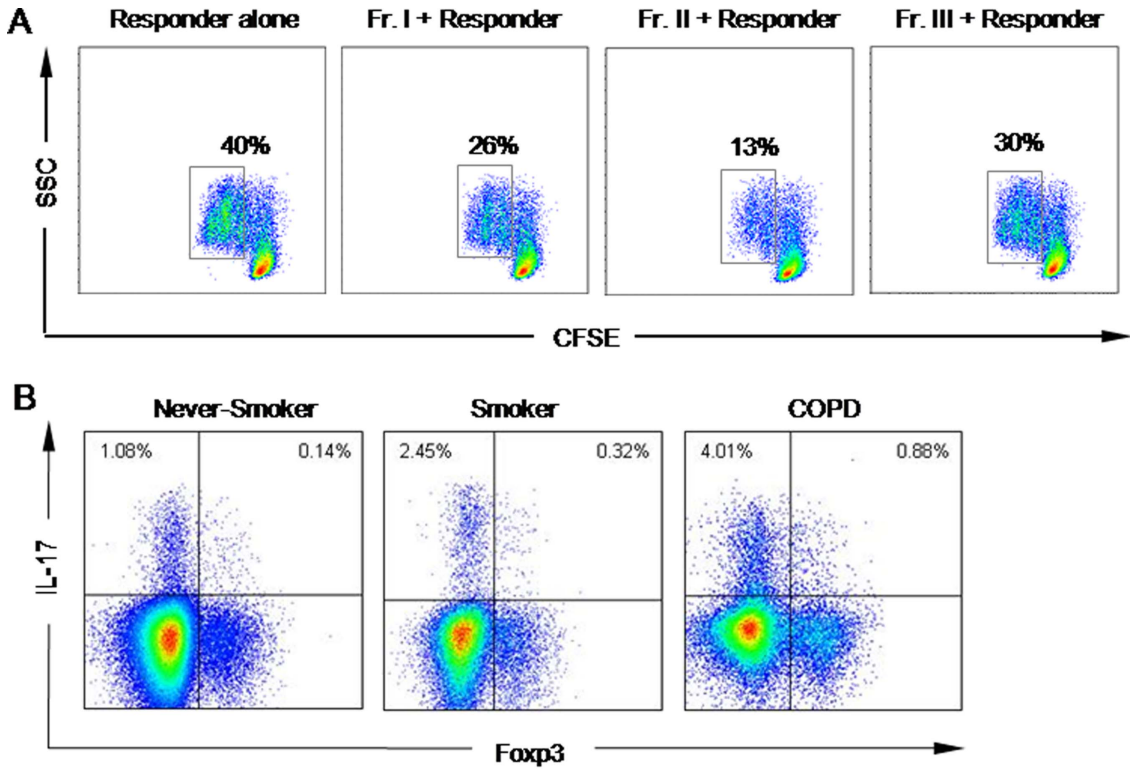

C
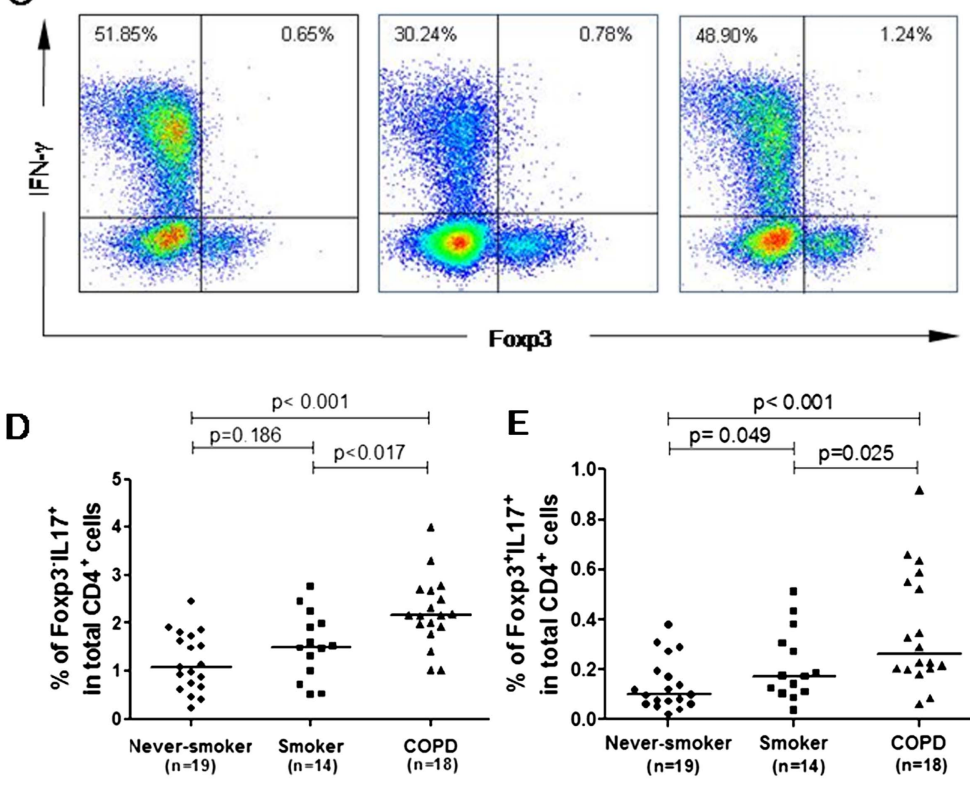

$\mathbf{F}$

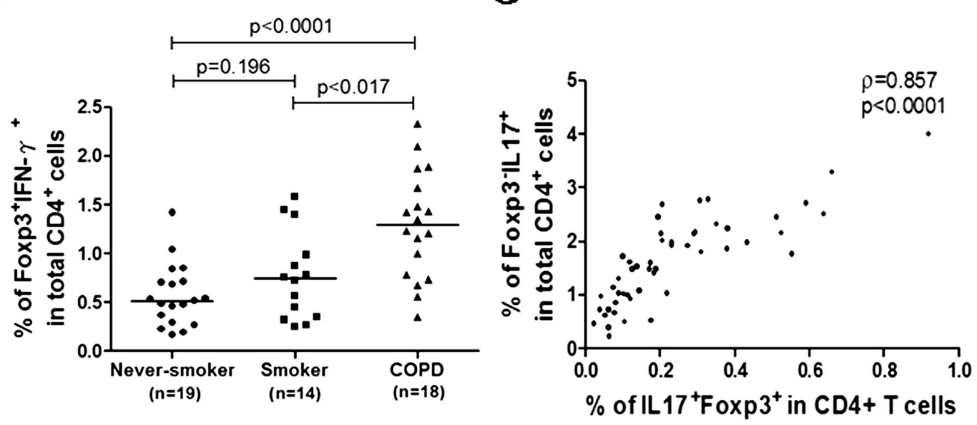

subpopulation in patients with COPD compared with smokers with normal lung function. More importantly, we linked the imbalance between these subsets with activation of CD8 T cells and the severity of COPD.

Despite a debate about their immune suppressive capacity, the inflammatory role of Fr III cells is well accepted due to their ability to produce IL-17 and IFN- $\gamma$. In smokers, we observed increased frequencies of three subpopulations, which suggested coexistence of anti-inflammatory and inflammatory responses. Strikingly, in comparison to smokers, patients with COPD exhibited increased proportions of Fr III cells and decreased proportions of rTregs and aTregs. This might reflect progression of inflammation and exhaustion of anti-inflammatory responses during disease progression. 
A

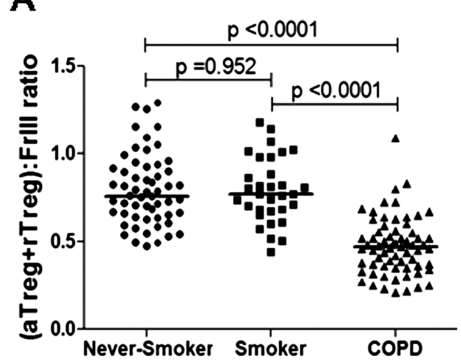

D

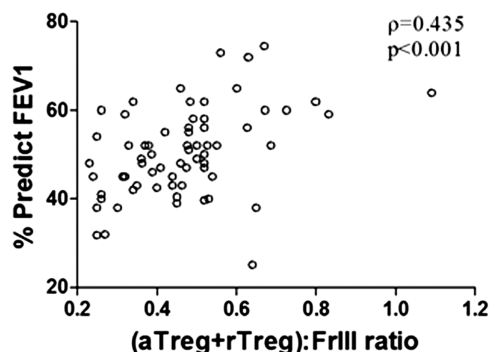

B

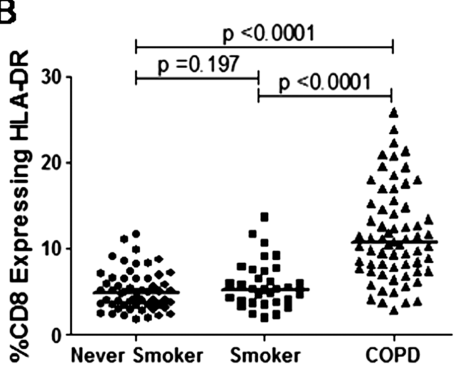

$\mathbf{E}$

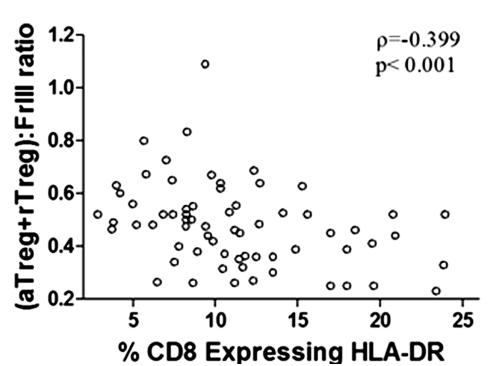

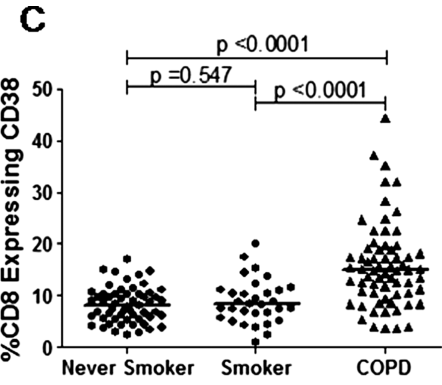

$\mathbf{F}$

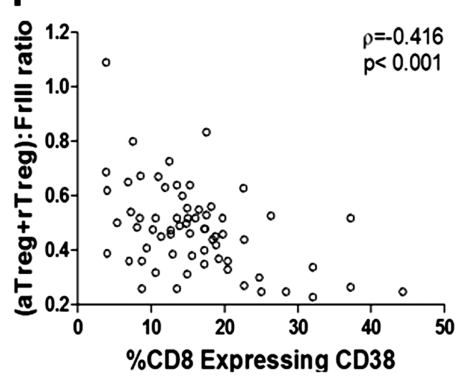

Figure 4 The imbalance between the suppressive and pro-inflammatory subpopulation correlated with disease severity and activation of CD8 T cells in the circulation. The (aTreg+rTreg):(Fr III) ratio was significantly decreased in patients with chronic obstructive pulmonary disease (COPD) compared with smokers and never-smokers (A); CD8 cells coexpressing HLA-DR (B) or CD38 (C) were increased in patients with COPD. A p value $<0.017$ was considered significant. The (aTreg+rTreg):(Fr III) ratio correlated with forced expiratory volume in $1 \mathrm{~s}\left(\mathrm{FEV}_{1}\right)$ predicted values (D) and CD8 T-lymphocyte activation status $(E, F)$ in patients with COPD. A p value $<0.05$ was considered statistically significant. Horizontal lines indicate median values. Fr I, CD25 ${ }^{++}$CD45RA ${ }^{+}$resting T-regulatory cells (rTreg); Fr II, $\mathrm{CD}^{2} 5^{++} \mathrm{CD}_{5} \mathrm{RA}^{-}$activated T-regulatory cells (aTreg); Fr III, CD25 ${ }^{++}$CD45RA ${ }^{-}$cytokine-secreting cells.

Moreover, we further described an imbalance between antiinflammatory and pro-inflammatory subsets by measuring the (aTreg+rTreg):(Fr III) ratio. Despite the elevation of three tested subsets in smokers with normal lung function, the (aTreg $+\mathrm{rTreg}$ ):(Fr III) ratio remained stable as in never-smokers, which indicated that immune response homeostasis remained intact, although inflammatory and anti-inflammatory mechanisms were activated. However, in patients with COPD, as indicated by a significantly decreased (aTreg+rTreg):(Fr III) ratio, the balance between subpopulations tilted in favour of the inflammatory response, which might have facilitated inflammation-related lung injury. More importantly, in line with a decreased (aTreg + rTreg):(Fr III) ratio, we found a positive correlation between the circulating (aTreg+rTreg):(Fr III) ratio and $\mathrm{FEV}_{1} \%$, which further linked the immune imbalance with airflow limitation in patients with COPD. The stronger correlation between the BAL (aTreg+rTreg):(Fr III) ratio and $\mathrm{FEV}_{1} \%$ provided more evidence to support this link. From these findings we hypothesise that a decrease in the immunosuppressive Treg populations, together with enhanced pro-inflammatory responses, induced by long-term exposure to inhalation of particles or gases, for example, tobacco smoking, ${ }^{18}$ lead to persistent airway inflammation dominated by CD8 T cells. This persistent airway inflammation is believed to be involved in the progressive loss of lung function characteristic of COPD.

It has been shown that CD8 $\mathrm{T}$ cells predominate over CD4 $\mathrm{T}$ cells in the airways and lung parenchyma of patients with COPD. ${ }^{15}$ COPD severity is correlated significantly with the frequency of activated CD8 $\mathrm{T}$ cells in peripheral blood. ${ }^{33}$ Moreover, previous studies have demonstrated opposing roles for Th17 cells and Tregs in CD8 cell activation: Treg cells suppress the proliferation of activated CD8 T cells via a variety of mechanisms, ${ }^{10-15}$ whereas IL-17-secreting cells promote CD8 cell activation by inducing expression of IL-6, IL-8 and intracellular adhesion molecule $1 .^{34} 35$ It has been shown that BAL samples from patients with COPD also exhibit a higher CD8 cell:Treg ratio than smokers. ${ }^{17}$ In this study, we revealed a negative correlation between the circulating (aTreg+rTreg):(Fr III) ratio and frequency of activated CD8 T cells, which indicated that such enhanced activation of CD8 T cells might have resulted from immune imbalance in patients with COPD. Moreover, circulating CD8 T cells in patients with COPD exhibited elevated frequencies of cells expressing CD38 and HLA-DR, two general markers for T-cell activation, but not a transient marker CD69, which further indicated that activation of CD8 T cells in patients with COPD was a result of chronic inflammation rather than acute activation. Interestingly, pulmonary CD8 $\mathrm{T}$ cells in patients with COPD exhibited elevated expression of CD69 and HLA-DR, suggesting ongoing activation of $\mathrm{T}$ cells at the site of inflammation.

In summary, this study provides further evidence for the role of adaptive immunity in COPD pathogenesis, and demonstrates that the balance between the subpopulations of previously described $\mathrm{CD}^{+}{ }^{+}$Foxp $^{+}$Tregs may contribute to the progression of inflammation in the lung. Of note, the correlations between the (aTreg+rTreg):Fr III ratio and activated CD8 $\mathrm{T}$ cells or $\mathrm{FEV}_{1} \%$ existed in BAL, and to a lesser extent, in peripheral blood, indicating a closer correlation between loss of lung function and local immune activation. Furthermore, our data extend local immune activation to systemic immune activation, which might facilitate our understanding of the underlying mechanisms for local and systemic inflammation in COPD. Future therapeutic strategies to control inflammatory responses in COPD may be directed at modulation of different subpopulations of $\mathrm{CD}^{+}$Foxp $^{+}$cells to restore immune homeostasis. 

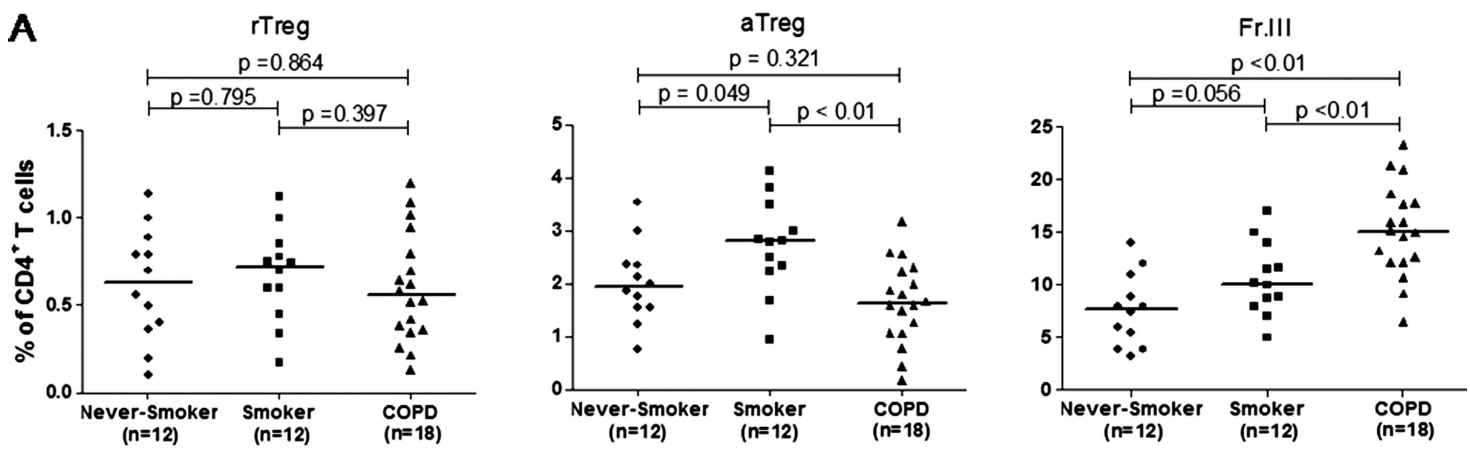

B

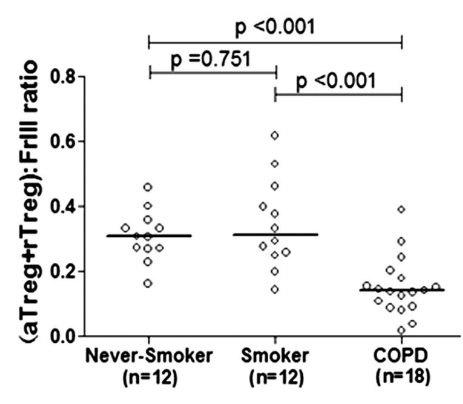

C

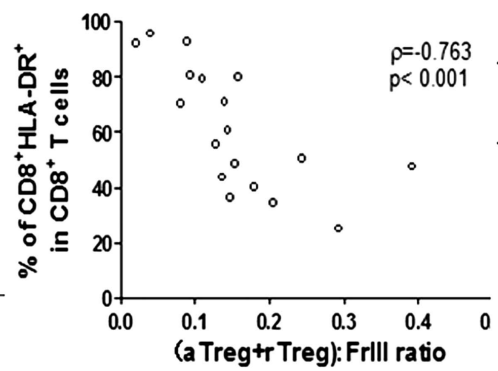

D

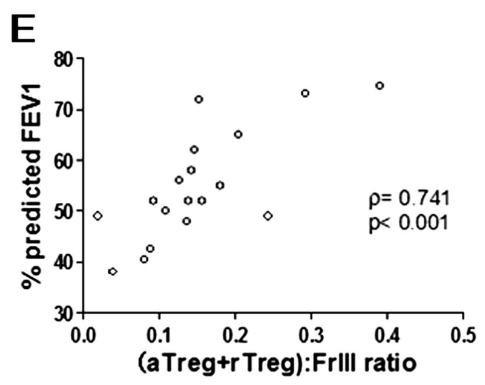

Figure 5 The percentages of each Foxp $3^{+}$subset among CD4 T cells (A) and (aTreg+rTreg):(Fr III) ratios (B) in the bronchoalveolar lavage of never-smokers, smokers and patients with chronic obstructive pulmonary disease (COPD). Horizontal lines indicate median values. A $p$ value $<0.017$ was considered significant. The (aTreg+rTreg):(Fr III) ratios correlated with CD8 T-lymphocyte activation status (C and D) and forced expiratory volume in $1 \mathrm{~s}\left(\mathrm{FEV}_{1}\right)$ predicted values $(\mathrm{E})$ in patients with COPD. A p value $<0.05$ was considered statistically significant. $\mathrm{Fr} \mathrm{I}_{\mathrm{l}} \mathrm{CD}^{2} 5^{++} \mathrm{CD} 45 \mathrm{RA} \mathrm{A}^{+}$ resting T-regulatory cells (rTreg); Fr II, CD25 ${ }^{+++}$CD45RA ${ }^{-}$activated T-regulatory cells (aTreg); Fr III, CD25 ${ }^{++}$CD45RA $^{-}$cytokine-secreting cells.

Acknowledgements The authors thank Husheng Zhang, Dongning Chen, Ran Li, Yang Wang, Li Li and Haiyan Sheng (Beijing Tongren Hospital) for their support with screening participating subjects throughout the study.

Contributors $\mathrm{JH}$ : contributed to recruiting the patients, performing all data collection, collecting and processing samples, and writing the manuscript. YS: contributed as primary investigator and was responsible for designing the study and writing the manuscript. YH and JH: performed laboratory-based assays. JZ, XL, PB and $\mathrm{XZ}$ : recruited the patients and collected clinical data. $\mathrm{HZ}$ : contributed as lead investigator and was responsible for designing the study, analysing the data and writing the manuscript. All authors read and approved the final manuscript.

Funding This work was supported by the Beijing Natural Science Foundation (No. 7092022), National Natural Science Foundation of China (No. 81170039) and 215 program (2009-2-13).

Competing interests None.

Ethics approval Ethics Committees, Beijing Tongren Hospital, Capital Medical University.

Provenance and peer review Not commissioned; externally peer reviewed.

\section{REFERENCES}

1 Hogg JC, Chu F, Utokaparch S, et al. The nature of small-airway obstruction in chronic obstructive pulmonary disease. N Engl J Med 2004;350:2645-53.

2 Nembrini C, Marsland BJ, Kopf M. IL-17-producing T cells in lung immunity and inflammation. J Allergy Clin Immunol 2009;123:986-94.
3 Doe C, Bafadhel M, Siddiqui S, et al. Expression of the Thelper 17-associated cytokines IL-17A and IL-17F in asthma and COPD. Chest 2010;138:1140-7.

4 Zhou L, Chong MM, Littman DR. Plasticity of $\mathrm{CD4}^{+} \mathrm{T}$ cell lineage differentiation. Immunity 2009;30:646-55.

5 Saetta M, Baraldo S, Corbino L, et al. CD8 ${ }^{+}$ve cells in the lungs of smokers with chronic obstructive pulmonary disease. Am J Respir Crit Care Med 1999;160:711-17.

6 Saetta M, Turato GB, Casoni GL, et al. Increased proportion of CD8 $8^{+} \mathrm{T}$ lymphocytes in the paratracheal lymph nodes of smokers with mild COPD. SarcoidosisVasc Lung Dis 2003;20:28-32.

7 Gan WQ, Man SF, Senthilselvan A, et al. Association between chronic obstructive pulmonary disease and systemic inflammation: a systematic review and a meta-analysis. Thorax 2004;59:574-80.

8 Monaco C, Andreakos E, Kiriakidis S, et al. T-cell mediated signalling in immune, inflammatory and angiogenic processes: the cascade of events leading to inflammatory diseases. Curr Drug Targets Inflamm Allergy 2004;3:35-42.

9 Curtis JL. Cell-mediated adaptive immune defense of the lungs. Proc Am Thorac Soc 2005;2:412-16

10 Lan RY, Ansari AA, Lian ZX, et al. Regulatory T cells: development, function and role in autoimmunity. Autoimmun Rev 2005;4:351-63.

11 Bluestone JA, Tang Q. How do CD4 ${ }^{+} \mathrm{CD} 25^{+}$regulatory T cells control autoimmunity? Curr Opin Immunol 2005;17:638-42.

12 Jiang $H$, Chess L. Regulation of immune responses by T cells. N Engl J Med 2006;354:1166-76

13 Ait-Oufella H, Salomon BL, Potteaux S, et al. Natural regulatory T cells control the development of atherosclerosis in mice. Nat Med 2006;12:178-80. 
14 Cederbom $\mathrm{L}$, Hall H, Ivars F. CD4 ${ }^{+} \mathrm{CD} 25^{+}$regulatory T cells down- regulate co-stimulatory molecules on antigen-presenting cells. Eur J Immunol 2000;30:1538-43.

15 Bonarius HP, Brandsma CA, Kerstjens HA, et al. Antinuclear autoantibodies are more prevalent in COPD in association with low body mass index but not with smoking history. Thorax 2011;66:101-7.

16 Feghali-Bostwick CA, Gadgil AS, Otterbein LE, et al. Autoantibodies in patients with chronic obstructive pulmonary disease. Am J Respir Crit Care Med 2008;177:156-63.

17 Smyth LJ, Starkey C, Vestbo J, et al. CD4-regulatory cells in COPD patients. Chest 2007:132:156-63.

18 Barceló B, Pons J, Ferrer JM, et al. Phenotypic characterisationof T-lymphocytes in COPD: abnormalCD4 ${ }^{+} \mathrm{CD} 25^{+}$regulatory T-lymphocyte response to tobacco smoking. Eur Respir J 2008;31:555-62.

19 Baraldo S, Saetta M. To reg or not to reg: that is the question in COPD. Eur Respir J 2008;31:486-8.

20 Isajevs S, Taivans I, Strazda G, et al. Decreased FOXP3 expression in small airways of smokers with COPD. Eur Respir J 2009;33:61-7.

21 Miyara M, Yoshioka Y, Kitoh A, et al. Functional delineation and differentiation dynamics of human $\mathrm{CD}^{+} \mathrm{T}$ cells expressing the FoxP3 transcription factor. Immunity 2009;30:899-911.

22 Simonetta F, Lecuroux C, Girault I, et al. Early and long-lasting alteration of effector CD45RA(-)Foxp3(high) regulatory T-cell homeostasis during HIV infection. J Infect Dis 2012;205:1510-19.

23 Marwaha AK, Crome SQ, Panagiotopoulos C, et al. Cutting edge: increased IL-17-secreting T cells in children with new-onset type 1 diabetes. J Immunol 2010;185:3814-18

24 Voo KS, Wang YH, Santori FR, et al. Identification of IL-17-producing FOXP3 ${ }^{+}$ regulatory T cells in humans. Proc Natl Acad Sci U S A 2009;106:4793-8.
25 Ayyoub M, Deknuydt F, Raimbaud I, et al. Human memory FOXP3 ${ }^{+}$Treg secrete IL-17 ex vivo and constitutively express the TH 17 lineage-specific transcription factor ROR $\gamma$ t. Proc Natl Acad Sci U S A 2009;106:8635-40.

26 Bettelli E, Oukka M, Kuchroo VK. T(H)-17 cells in the circle of immunity and autoimmunity. Nat Immunol 2007:8:345-50.

27 Vanaudenaerde BM, Verleden SE, Vos R, et al. Innate and adaptive interleukin-17-producing lymphocytes in chronic inflammatory lung disorders. Am J RespirCrit Care Med 2011;183:977-86.

28 Rabe KF, Hurd S, Anzueto A, et al. Global strategy for the diagnosis, management, and prevention of chronic obstructive pulmonary disease: GOLD executive summary. Am J Respir Crit Care Med 2007;176:532-55.

29 Tanino M, Betsuyaku T, Takeyabu K, et al. Increased levels of interleukin-8 in BAL fluid from smokers susceptible to pulmonary emphysema. Thorax 2002;57:405-11.

30 Zhong N, Wang C, Yao W, et al. Prevalence of chronic obstructive pulmonary disease in China. Am J Respir Crit Care Med 2007;176:753-60.

31 Kimmig S, Przybylski GK, Schmidt CA, et al. Two subsets of naive T helper cells with distinct $\mathrm{T}$ cell receptor excision circle content in human adult peripheral blood. J Exp Med 2002;195:789-94

32 Fontenot JD, Rudensky AY. A well adapted regulatory contrivance: regulatory T cell development and the forkhead family transcription factor Foxp3. Nat Immunol 2005;6:331-7.

33 Zhu X, Gadgil AS, Givelber R, et al. Peripheral T cell functions correlate with the severity of chronic obstructive pulmonary disease. J Immunol 2009;182:3270-7.

34 Yao Z, Painter SL, Fanslow WC, et al. Human IL-17: a novel cytokine derived from T cells. J Immunol 1995;155:5483-6.

35 Deeths MJ, Mescher MF. ICAM-1 and B7-1 provide similar but distinct costimulation for $\mathrm{CD}^{+} \mathrm{T}$ cells, while $\mathrm{CD} 4^{+} \mathrm{T}$ cells are poorly costimulated by ICAM. Eur J Immunol 1999;29:45-53. 